\title{
ON THE ASYMPTOTIC BEHAVIOR OF THE FOURIER TRANSFORM OF THE INDICATOR FUNCTION OF A CONVEX SET
}

\author{
BY \\ BURTON RANDOL
}

Suppose $C$ is a compact, convex subset of $R^{n}$, having a smooth boundary $\partial C$. Let $F(r, \theta)$ be the Fourier transform, in polar coordinates $\left(r=\left(x_{1}^{2}+\cdots+x_{n}^{2}\right)^{1 / 2}\right.$; $\left.\theta=\left(x_{1} / r, \ldots, x_{n} / r\right)\right)$ of the indicator function of the set $C$, where by the indicator function of $C$, we mean the function whose value on $C$ is 1 , and whose value on the complement of $C$ is 0 . Then it is known (cf. [1], [2]) that the function $\Phi(\theta)$ $=\sup _{r} r^{(n+1) / 2}|F(r, \theta)|$ is bounded on $S^{n-1}$, provided $\partial C$ is sufficiently smooth, and has everywhere positive Gaussian curvature. If $\partial C$ has points of zero curvature, this need no longer be true (cf. [4]). The following, however, remains true.

THEOREM 1. If $C$ is compact and convex, and $\partial C$ is analytic, then for some $p>2$, $\Phi(\theta)$ is of class $L^{p}$ on $S^{n-1}$.

This is a consequence of the following stronger result.

TheOREM 2. Suppose $C$ is compact and convex, and $\partial C$ is analytic. Let $N(\theta)$ be the map which takes $S^{n-1}$ homeomorphically onto $\partial C$, by sending $\theta \in S^{n-1}$ into the unique point on $\partial C$ at which the exterior normal to $\partial C$ has direction $\theta .(N(\theta)$ is the inverse of the Gaussian normal map. The fact that $N(\theta)$ is 1-1 follows immediately from the convexity of $C$, and the analyticity of $\partial C$.) For a point $\alpha \in \partial C$, let $K(\alpha)$ be the Gaussian curvature of $\partial C$ at $\alpha$. Then there exist three $C^{\infty}$ functions $B_{1}(r, \theta), B_{2}(r, \theta)$, and $R(r, \theta)$, such that

(1) $F(r, \theta)=B_{1}(r, \theta)+B_{2}(r, \theta)+R(r, \theta)$.

(2) For some $M>0$,

$$
\left|B_{1}(r, \theta)\right| \leqq M[K(N(\theta))]^{-1 / 2} r^{-(n+1) / 2},
$$

and

$$
\left|B_{2}(r, \theta)\right| \leqq M[K(N(-\theta))]^{-1 / 2} r^{-(n+1) / 2} .
$$

(3) For any fixed $k, R(r, \theta)=O\left(r^{-k}\right)$, uniformly in $\theta$.

Proof of Theorem 1 (assuming Theorem 2). Suppose Theorem 2 has been established. Then to prove Theorem 1 , it suffices to show that $[K(N(\theta))]^{-1 / 2}$ and

Received by the editors February 26, 1968. 
$[K(N(-\theta))]^{-1 / 2}$ are both in $L^{p}\left(S^{n-1}\right)$, for some $p>2$. In order to show that $[K(N(\theta))]^{-1 / 2}$ is in $L^{p}\left(S^{n-1}\right)$ for some $p>2$, it clearly suffices to show that $J_{1}(\alpha)[K(\alpha)]^{-p / 2}$ is of class $L^{1}$ on $\partial C$, where $J_{1}(\alpha)$ is the Radon-Nikodym derivative of the inverse of $N(\theta)$. Similarly, to show that $[K(N(-\theta))]^{-1 / 2}$ is in $L^{p}\left(S^{n-1}\right)$, it clearly suffices to show that $J_{2}(\alpha)[K(\alpha)]^{-p / 2}$ is of class $L^{1}$ on $\partial C$, where $J_{2}(\alpha)$ is the RadonNikodym derivative of the inverse of the composition of $N(\theta)$ with the antipodal map of $S^{n-1}$ onto itself. Since $J_{1}(\alpha)=J_{2}(\alpha)=K(\alpha)$, the desired result is a consequence of the following lemma.

Lemma 1. Suppose $f(Y) \not \equiv 0$ is real analytic in a neighborhood of the origin in $R^{n}$. Then there exists a neighborhood $N$ of the origin, such that for sufficiently small $\delta>0,|f(Y)|^{-\delta}$ is integrable over $N$.

Proof. We may assume $f(0)=0$. Now it follows from the Weierstrass preparation theorem, that in a suitable orthonormal coordinate system $(y, t)=\left(y_{1}, \ldots, y_{n-1}, t\right)$, $f(y, t)$ can, for small $y$ and $t$, be expressed in the form $\Omega(y, t) P(y, t)$, where $P(y, t)=\left(t^{k}+H_{k-1}(y) t^{k-1}+\cdots+H_{1}(y) t+H_{0}(y)\right)$, with $k$ a positive integer and the $H_{j}$ 's analytic, and $\Omega(y, t)$ is analytic and bounded away from zero. Since $\Omega(y, t)$ is bounded away from zero, it suffices to prove the lemma for $P(y, t)$. Now $P(y, t)$ $=\left(t-a_{1}\right) \cdots\left(t-a_{k}\right)$, for suitable $a_{j}$ 's, which depend, of course, on $y$. Suppose now $\varepsilon>0$ is fixed, and $\lambda \in[0,1)$. Then

$$
\int_{-\varepsilon}^{\varepsilon}\left|\left(t-a_{1}\right) \cdots\left(t-a_{k}\right)\right|^{-\lambda / k} d t \leqq \frac{1}{k} \sum_{j=1}^{k} \int_{-\varepsilon}^{\varepsilon}\left|t-a_{j}\right|^{-\lambda} d t,
$$

by the inequality between the geometric and arithmetic means. Since $\int_{-\varepsilon}^{\varepsilon}|t-z|^{-\lambda} d t$ is uniformly bounded for all complex $z$, the desired result follows from Fubini's theorem, if we restrict our attention to a small hypercube about the origin, take $\delta<1 / k$, and integrate first in the $t$-direction.

Proof of Theorem 2. By definition,

$$
F(r, \theta)=\int_{C} \exp (2 \pi i r(\theta, Y)) d V_{Y},
$$

where $d V_{Y}$ is the volume element on $R^{n}$. By the divergence theorem, the last integral equals

$$
\frac{1}{2 \pi i r} \int_{\partial C} \exp (2 \pi i r(\theta, Y))(\theta, n(Y)) d S_{Y},
$$

where $d S_{Y}$ is the area element on $\partial C$, and $n(Y)$ is the exterior normal to $\partial C$ at the point $Y$. It is evident that for a given $\theta$, the function $(\theta, n(Y))$ is analytic on $\partial C$. Suppose now that $\varepsilon>0$, and let $H(Y)$ be a nonnegative $C^{\infty}$ function in $R^{n}$, which is supported in the ball $|Y| \leqq \varepsilon$, and which is identically 1 in a neighborhood of the origin. For each $\theta \in S^{n-1}$, introduce functions $h_{1}(\theta, Y)$ and $h_{2}(\theta, Y)$ on $\partial C$, by 
defining $h_{1}(\theta, Y)$ to be the restriction to $\partial C$ of $H(Y-N(\theta))$, and $h_{2}(\theta, Y)$ to be the restriction to $\partial C$ of $H(Y-N(-\theta))$. Then if $\varepsilon$ is sufficiently small, (1) equals

$$
\begin{aligned}
\frac{1}{2 \pi i r} \int_{\partial C} \exp (2 \pi i r(\theta, Y)) h_{1}(\theta, Y)(\theta, n(Y)) d S_{Y} \\
\quad+\frac{1}{2 \pi i r} \int_{\partial C} \exp (2 \pi i r(\theta, Y)) h_{2}(\theta, Y)(\theta, n(Y)) d S_{Y}+R(r, \theta),
\end{aligned}
$$

where $R(r, \theta)=O\left(r^{-k}\right)$ uniformly in $\theta$, for any fixed $k$ (cf. [3, p. 767]).

It thus suffices to show that the integrals in (2) are bounded by multiples of $[K(N(\theta))]^{-1 / 2} r^{-(n+1) / 2}$, and $[K(N(-\theta))]^{-1 / 2} r^{-(n+1) / 2}$, respectively. We shall prove this for the first integral. The proof for the second integral proceeds along the same lines.

Introduce polar coordinates $(\rho, \phi)$ in the $(n-1)$-dimensional hyperplane tangent to $\partial C$ at $N(\theta)$, placing the origin at $N(\theta)$. Then

$$
\begin{aligned}
\int_{\partial C} \exp (2 \pi i r(\theta, Y)) h_{1}(\theta, Y)(\theta, n(Y)) d S_{Y} \\
=\int_{S^{n-2}} d S_{\phi} \int_{0}^{\varepsilon} \rho^{n-2} f(\theta, \phi, \rho) \exp (2 \pi i r E(\theta, \phi, \rho)) d \rho,
\end{aligned}
$$

where $f(\theta, \phi, \rho)$ is, for each $\theta, C^{\infty}$ with support in the ball $|\rho| \leqq \varepsilon$, and the partial derivatives of $f(\theta, \phi, \rho)$ with respect to $\rho$ can be bounded independently of $\theta$ and $\phi$. $E(\theta, \phi, \rho)$ is of the form $d(\theta)-\psi(\theta, \phi, \rho)$, where $d(\theta)$ is the distance from the origin in $R^{n}$ to the support plane to $\partial C$ at $N(\theta)$, and $\psi(\theta, \phi, \rho)$ may be assumed, after an orthogonal transformation of the $(\rho, \phi)$-coordinate system if necessary, to be of the form $\frac{1}{2}\left[k_{1}(\theta) \phi_{1}^{2}+\cdots+k_{n-1}(\theta) \phi_{n-1}^{2}\right]_{\rho}^{2}+O\left(\rho^{3}\right)$, where $\left(\phi_{1}, \ldots, \phi_{n-1}\right)=\phi$, and the $k_{i}$ 's are the principal curvatures of $\partial C$ at $N(\theta)$.

The following lemma shows that Theorem 2 will be proved if we can show that

$$
\begin{aligned}
& \int_{0}^{\varepsilon} \rho^{n-2} f(\theta, \phi, \rho) \exp (2 \pi i r E(\theta, \phi, \rho)) d \rho \\
& \leqq M\left[k_{1}(\theta) \phi_{1}^{2}+\cdots+k_{n-1}(\theta) \phi_{n-1}^{2}\right]^{-(n-1) / 2} r^{-(n-1) / 2},
\end{aligned}
$$

for some $M>0$.

LEMMA 2.

$$
\begin{aligned}
\int_{S^{n-2}}\left[k_{1}(\theta) \phi_{1}^{2}+\cdots+k_{n-1}(\theta) \phi_{n-1}^{2}\right]^{-(n-1) / 2} d S_{\phi} & =\frac{2 \pi^{(n-1) / 2}}{\Gamma((n-1) / 2)}\left(k_{1}(\theta) \cdots k_{n-1}(\theta)\right)^{-1 / 2} \\
& =\frac{2 \pi^{(n-1) / 2}}{\Gamma((n-1) / 2)}[K(N(\theta))]^{-1 / 2}
\end{aligned}
$$

Proof. Consider the integral

$$
\begin{aligned}
\int_{-\infty}^{\infty} d y_{n-1} \cdots \int_{-\infty}^{\infty} \exp \left\{-\left(k_{1}(\theta) y_{1}^{2}+\cdots+k_{n-1}(\theta) y_{n-1}^{2}\right)\right\} d y_{1} \\
=\pi^{(n-1) / 2}\left(k_{1}(\theta) \cdots k_{n-1}(\theta)\right)^{-1 / 2} .
\end{aligned}
$$


In polar coordinates, the integral becomes

$$
\int_{S^{n-2}} d S_{\phi} \int_{0}^{\infty} \rho^{n-2} \exp \left\{-\left(k_{1}(\theta) \phi_{1}^{2}+\cdots+k_{n-1}(\theta) \phi_{n-1}^{2}\right)\right\} d \rho .
$$

The inner integral is a Mellin transform, whose value is

$$
\frac{1}{2} \Gamma((n-1) / 2)\left(k_{1}(\theta) \phi_{1}^{2}+\cdots+k_{n-1}(\theta) \phi_{n-1}^{2}\right)^{-(n-1) / 2},
$$

and the result follows immediately.

In order to prove (3), we need some information about the derivatives with respect to $\rho$ of $E(\theta, \phi, \rho)$. Note, to begin with, that for each $\theta, E(\theta, \phi, \rho)$ is, for small $\rho$, of the form $d(\theta)-\sum_{n=2}^{\infty} a_{n}(\theta, \phi) \rho^{n}$, where $a_{2}(\theta, \phi)=\frac{1}{2}\left[k_{1}(\theta) \phi_{1}^{2}+\cdots+\right.$ $\left.k_{n-1}(\theta) \phi_{n-1}^{2}\right]$. Moreover, it is evident from compactness arguments that

(1) There exists an integer $N$, and a positive number $\mu$, such that

$$
\sum_{n=2}^{N}\left|a_{n}(\theta, \phi)\right| \geqq \mu, \quad \text { for all }(\theta, \phi) \in S^{n-1} \times S^{n-2} \text {. }
$$

(2) There exists some fixed power series $\sum_{n=2}^{\infty} b_{n} \rho^{n}$, having a positive radius of convergence, such that $\left|a_{n}(\theta, \phi)\right| \leqq\left|b_{n}\right|$, for all $(\theta, \phi) \in S^{n-1} \times S^{n-2}$. (To see this, we could, for example, argue as follows: it follows easily from the Heine-Borel theorem, that there exists some $\delta>0$, such that for all $(\theta, \phi) \in S^{n-1} \times S^{n-2}$, the function $\sum_{n=2}^{\infty} a_{n}(\theta, \phi) \rho^{n}$ has a complex analytic extension into a neighborhood of the disk $|\rho| \leqq \delta$. Moreover, if $\delta$ is small enough, the function $\max _{|\rho|=\delta}\left|\sum_{n=2}^{\infty} a_{n}(\theta, \phi) \rho^{n}\right|$ is a continuous function of $\theta$ and $\phi$, and hence is bounded, say by $M$, on $S^{n-1}$ $\times S^{n-2}$. By Cauchy's estimate, $\left|a_{n}(\theta, \phi)\right| \leqq M \delta^{-n}$.)

LEMMA 3. For a polynomial $\pi(x)=a_{m} x^{m}+\cdots+a_{1} x+a_{0}$, with real or complex coefficients, define, for $0<r \leqq 1, M_{\pi}(r)=\max _{0 \leqq x \leqq r}|\pi(x)|$, and $M_{\pi}^{*}(r)=\left|a_{m}\right| r^{m}+\cdots$ $+\left|a_{1}\right| r+\left|a_{0}\right|$. Then there exists a constant $M>0$, depending on $m$, but otherwise independent of $\pi(x)$, such that $M_{\pi}(r) \geqq M M_{\pi}^{*}(r)$, for $0<r \leqq 1$.

Proof. By the change of variable $x_{1}=x / r$, we may assume $r=1$. Now $M_{\pi}(1)$ and $M_{\pi}^{*}(1)$ are both norms on $C^{m+1}$, if we identify the coefficients of $\pi$ with coordinates in $C^{m+1}$. The result thus follows from the equivalence of norms on $C^{m+1}$.

Lemma 4. Define $E_{*}(\theta, \phi, \rho)=\sum_{n=2}^{\infty}\left|a_{n}(\theta, \phi)\right| \rho^{n}$, and denote by $E^{(j)}(\theta, \phi, \rho)$ and $E_{*}^{(j)}(\theta, \phi, \rho)$, respectively, the jth partial derivatives with respect to $\rho$ of $E(\theta, \phi, \rho)$ and $E_{*}(\theta, \phi, \rho)$. Then there exist positive numbers $M$ and $\delta$, such that $\left|E^{(1)}(\theta, \phi, \rho)\right|$ $\geqq M E_{*}^{(1)}(\theta, \phi, \rho)$, for $0 \leqq \rho \leqq \delta$, and $(\theta, \phi) \in \delta^{n-1} \times \delta^{n-2}$.

Proof. We know that there exists a number $\mu>0$, and an integer $N$, such that $\sum_{n=2}^{N}\left|a_{n}(\theta, \phi)\right| \geqq \mu$, for all $\theta$ and $\phi$. Moreover, there exists a power series $\sum_{n=2}^{\infty} b_{n} \rho^{n}$, having a positive radius of convergence, such that $\left|a_{n}(\theta, \phi)\right| \leqq\left|b_{n}\right|$, for all $\theta$ and $\phi$. 
Define $\pi(\theta, \phi, \rho)=\sum_{n=2}^{N} n a_{n}(\theta, \phi) \rho^{n-1}, \pi^{*}(\theta, \phi, \rho)=\sum_{n=2}^{N} n\left|a_{n}(\theta, \phi)\right| \rho^{n-1}$, and

$$
M_{\pi}(\theta, \phi, \rho)=\max _{0 \leqq t \leqq \rho}|\pi(\theta, \phi, t)| \text {. }
$$

Then $E_{*}^{(1)}(\theta, \phi, \rho)=\pi^{*}(\theta, \phi, \rho)+o\left(\pi^{*}(\theta, \phi, \rho)\right)$, and

$$
\left|E^{(1)}(\theta, \phi, \rho)\right|=\left|\pi(\theta, \phi, \rho)+o\left(\pi^{*}(\theta, \phi, \rho)\right)\right|,
$$

where the " $o$ " terms are of uniformly lower order for $(\theta, \phi) \in S^{n-1} \times S^{n-2}$. Now $\left|E^{(1)}(\theta, \phi, \rho)\right|$ is, for each $\theta$ and $\phi$, an increasing function of $\rho$, since $C$ is convex, and this implies that $\left|E^{(1)}(\theta, \phi, \rho)\right| \geqq\left|M_{\pi}(\theta, \phi, \rho)+o\left(\pi^{*}(\theta, \phi, \rho)\right)\right|$. By Lemma 3, the last inequality implies that $\left|E^{(1)}(\theta, \phi, \rho)\right| \geqq M^{\prime}\left(\pi^{*}(\theta, \phi, \rho)+o\left(\pi^{*}(\theta, \phi, \rho)\right)\right)$, for some $M^{\prime}>0$, and this proves the desired result.

Corollary. $\left|E^{(1)}(\theta, \phi, \rho)\right| \geqq\left|a_{2}(\theta, \phi)\right| \rho=\frac{1}{2}\left[k_{1}(\theta) \phi_{1}^{2}+\cdots+k_{n-1}(\theta) \phi_{n-1}^{2}\right] \rho$.

Lemma 5. Suppose $m$ is a fixed positive integer. Then there exist numbers $M$, $\delta>0$, such that for $j=1, \ldots, m, 0 \leqq \rho \leqq \delta$, and $(\theta, \phi) \in S^{n-1} \times S^{n-2}$,

$$
\rho^{j-1}\left|E^{(j)}(\theta, \phi, \rho)\right| \leqq M\left|E^{(1)}(\theta, \phi, \rho)\right| \text {. }
$$

Proof. Define $\pi^{*}(\theta, \phi, \rho)$ as in the proof of Lemma 4. Then it is clear from power series considerations, that for any integer $m>0$, there exists a number $M^{\prime}>0$, such that $\rho^{j-1} E_{*}^{(j)}(\theta, \phi, \rho) \leqq M^{\prime} \pi^{*}(\theta, \phi, \rho)$, for sufficiently small $\rho$. The desired conclusion thus follows from Lemma 4.

In order to simplify the proof of (3), we next introduce the following functions, which occur when we integrate the left side of (3) by parts:

$$
\begin{aligned}
& L_{1}(\theta, \phi, \rho)=\frac{\rho^{n-2} f(\theta, \phi, \rho)}{E^{(1)}(\theta, \phi, \rho)}, \\
& L_{k}(\theta, \phi, \rho)=\frac{(\partial / \partial \rho)\left(L_{k-1}(\theta, \phi, \rho)\right)}{E^{(1)}(\theta, \phi, \rho)}, \text { for } k \geqq 2 .
\end{aligned}
$$

LEMMA 6. For any integer $j \geqq 1$, there exists a $\delta>0$, such that for $\rho \in[0, \delta]$,

(a) $L_{j}(\theta, \phi, \rho)=O\left(\left[k_{1}(\theta) \phi_{1}^{2}+\cdots+k_{n-1}(\theta) \phi_{n-1}\right]^{-j} \rho^{n-1-2 j}\right)$,

(b) $(\partial / \partial \rho) L_{j}(\theta, \phi, \rho)=O\left(\left[k_{1}(\theta) \phi_{1}^{2}+\cdots+k_{n-1}(\theta) \phi_{n-1}^{2}\right]^{-j} \rho^{n-2-2 \jmath}\right)$, uniformly on $S^{n-1} \times S^{n-2}$.

Proof. This follows from Lemma 5 and the corollary to Lemma 4 in the following way:

For any given $j, L_{j}$ can be expressed in the form $\sum_{i} \lambda_{i} \rho^{u_{i}}\left(E^{(1)}\right)^{-v_{i}}$, where the $\lambda_{i}$ 's are products of various derivatives with respect to $\rho$ of $f$ and $E^{(1)}$, and the $u_{i}$ 's and $v_{i}$ 's are appropriate integers. Now

$$
\begin{aligned}
\frac{\partial}{\partial \rho} L_{j} & =\frac{\partial}{\partial \rho}\left[\sum_{i} \lambda_{i} \rho^{u_{i}}\left(E^{(1)}\right)^{-v_{i}}\right] \\
& =\sum_{i}\left\{\rho^{u_{i}}\left(E^{(1)}\right)^{-v_{i}} \frac{\partial \lambda_{i}}{\partial \rho}+u_{i} \lambda_{i} \rho^{u_{i}-1}\left(E^{(1)}\right)^{-v_{i}}-v_{i} \lambda_{i} \rho^{u_{i}} E^{(2)}\left(E^{(1)}\right)^{-\left(v_{i}+1\right)}\right\}
\end{aligned}
$$

and from this it is evident, by Lemma 5 , that $\partial L_{j} / \partial \rho$ can be bounded by $\rho^{-1}$ times a multiple of a bound for $L_{j}$. Similarly, by the corollary to Lemma $4, L_{j}=\left(E^{(1)}\right)^{-1}$ . $\partial L_{j-1} / \partial \rho$ can be bounded by $\rho^{-2}\left[k_{1}(\theta) \phi_{1}^{2}+\cdots+k_{n-1}(\theta) \phi_{n-1}^{2}\right]^{-1 / 2}$ times a multiple 
of a bound for $L_{j-1}$. The conclusions of Lemma 6 thus follow from an obvious induction procedure.

We shall now establish (3), and we shall assume, in what follows, that the $\varepsilon$ which occurs in (3) is small enough so that the conclusions of Lemma 6 are valid for $\rho \in[0, \varepsilon]$, and $j \leqq[(n+1) / 2]$. We may also assume that $K(N(\theta))>0$. We begin with the case in which $n$ is even. Integrating by parts $(n-2) / 2$ times, and using the fact that $E^{(1)}(\theta, \phi, \rho)$ has a first order zero in $\rho$ (since $\left.K(N(\theta))>0\right)$, and that $f(\theta, \phi, \rho)$ and all its derivatives with respect to $\rho$ vanish at $\rho=\varepsilon$, the left side of (3) takes the form

$$
\frac{\text { const. }}{r^{(n-2) / 2}} \int_{0}^{\varepsilon}\left(\frac{\partial}{\partial \rho} L_{(n-2) / 2}\right) \exp (2 \pi i r E) d \rho .
$$

Now define $\delta=\left[k_{1}(\theta) \phi_{1}^{2}+\cdots+k_{n-1}(\theta) \phi_{n-1}^{2}\right]^{-1 / 2} r^{-1 / 2}$. Then $\int_{0}^{\varepsilon}=\int_{0}^{\delta}+\int_{\delta}^{\varepsilon}$.

By Lemma 6, the first integral is bounded by a multiple of

$$
\left[k_{1}(\theta) \phi_{1}^{2}+\cdots+k_{n-1}(\theta) \phi_{n-1}^{2}\right]^{-(n-1) / 2} r^{-1 / 2} .
$$

The second integral, after one integration by parts, can be expressed in the form

$$
\left.(2 \pi i r)^{-1} L_{n / 2} \exp (2 \pi i r E)\right]_{\rho=\delta}^{\varepsilon}-(2 \pi i r)^{-1} \int_{\delta}^{\varepsilon}\left(\frac{\partial}{\partial \rho} L_{n / 2}\right) \exp (2 \pi i r E) d \rho
$$

and it follows from Lemma 6 that both these terms are bounded by a multiple of $\left[k_{1}(\theta) \phi_{1}^{2}+\cdots+k_{n-1}(\theta) \phi_{n-1}^{2}\right]^{-(n-1) / 2} r^{-1 / 2}$, which proves Theorem 2 if $n$ is even.

If $n$ is odd, we integrate the left side of (3) $(n-3) / 2$ times by parts, to obtain an expression of the form

$$
\frac{\text { const. }}{r^{(n-3) / 2}} \int_{0}^{\varepsilon}\left(\frac{\partial}{\partial \rho} L_{(n-3) / 2}\right) \exp (2 \pi i r E) d \rho
$$

Now define $\delta$ as before, and again note that $\int_{0}^{\varepsilon}=\int_{0}^{\delta}+\int_{\delta}^{\varepsilon}$.

By Lemma 6, the first integral is bounded by a multiple of

$$
\left[k_{1}(\theta) \phi_{1}^{2}+\cdots+k_{n-1}(\theta) \phi_{n-1}^{2}\right]^{-(n-1) / 2} r^{-1} .
$$

The second integral, after integration by parts, takes the form

$$
\left.(2 \pi i r)^{-1} L_{(n-1) / 2} \exp (2 \pi i r E)\right]_{\rho=\delta}^{\varepsilon}-(2 \pi i r)^{-1} \int_{\delta}^{\varepsilon}\left(\frac{\partial}{\partial \rho} L_{(n-1) / 2}\right) \exp (2 \pi i r E) d \rho .
$$

By Lemma 6, the first quantity is bounded by a multiple of

$$
\left[k_{1}(\theta) \phi_{1}^{2}+\cdots+k_{n-1}(\theta) \phi_{n-1}^{2}\right]^{-(n-1) / 2} r^{-1} .
$$

If, now, we integrate the remaining quantity once more by parts, we obtain

$$
\left.-(2 \pi i r)^{-2} L_{(n+1) / 2} \exp (2 \pi i r E)\right]_{\rho=\delta}^{\varepsilon}+(2 \pi i r)^{-2} \int\left(\frac{\partial}{\partial \rho} L_{(n+1) / 2}\right) \exp (2 \pi i r E) d \rho,
$$

and it follows from Lemma 6 that both these quantities are bounded by a multiple of $\left[k_{1}(\theta) \phi_{1}^{2}+\cdots+k_{n-1}(\theta) \phi_{n-1}^{2}\right]^{-(n-1) / 2} r^{-1}$, which completes the proof of Theorem 2. 
Remarks. 1. It is, I believe, quite likely that Theorem 1 remains true without the convexity requirement on $C$. This is, at any rate, certainly true in two dimensions (cf. [5]).

2. Theorem 1 has applications to the geometry of numbers. We mention two here. The proofs are straightforward generalizations of the methods in [5].

(A) Suppose $C$ is compact and convex, and $\partial C$ is analytic. Denote by $V$ the volume of $C$. For $s \in S O(n)$, let $L_{s}$ be the image of the integral lattice-points under $s$. For $x>0$, let $N(x, s)$ be the number of points in $L_{s}$ which intersect the set $x C$, and let $R(x, s)=N(x, s)-V x^{n}$. Then

$$
\int_{S O(n)}|R(x, s)| d s=O\left(x^{n(n-1) /(n+1)}\right),
$$

where $d s$ is normalized Haar measure on $S O(n)$.

(B) Let $C$ be as in (A). Let $G$ be the group of all motions of the form $s t$, where $s \in S O(n)$ and $t$ is a translation. Let $I$ be the subgroup of integral translations, and define $H=G / I$. Then $H$ clearly acts on the set of integral lattice-points in a welldefined way. For $h \in H$, let $L_{h}$ be the image of the integral lattice-points under $h$, and let $N(x, h)$ be the number of points in $L_{h}$ which intersect the set $x C$. Define $R(x, h)=N(x, h)-V x^{n}$. Then

$$
\left(\int_{H}|R(x, h)|^{2} d h\right)^{1 / 2}=O\left(x^{(n-1) / 2}\right)
$$

where $d h$ is normalized Haar measure on $H$.

\section{REFERENCES}

1. C. S. Herz, Fourier transforms related to convex sets, Ann. of Math. 75 (1962), 81-92.

2. E. Hlawka, Uber Integrale auf konvexen Körpern. I, Monatsh. Math. 54 (1950), 1-36.

3. Walter Littman, Fourier transforms of surface-carried measures and differentiability of surface averages, Bull. Amer. Math. Soc. 69 (1963), 766-770.

4. Burton Randol, A lattice-point problem, Trans. Amer. Math. Soc. 121 (1966), 257-268.

5. - On the Fourier transform of the indicator function of a planar set, Trans. Amer. Math. Soc. 139 (1969), 271-278

YALE UNIVERSITY,

New Haven, Connecticut 